\title{
Early Detection of Gestational Diabetes Mellitus (GDM) by Measuring Maternal Serum Uric Acid in the First Trimester of Pregnancy
}

\author{
Ali El- Shabrawy Ali, Hend Salah Abdo, Ahmed El- Sayed Mansour and Marwah Fathy Abdalla \\ Department of Obstetrics and Gynecology, Faculty of Medicine - Zagazig University \\ *Corresponding author: Marwah Fathy Abdalla; Mobile: 01003967044; E-mail: dr.marwah.fathy@ hotmail.com
}

\begin{abstract}
Background: Gestational diabetes mellitus (GDM) is a common condition with risks for mother and baby. Type 2 diabetes mellitus (T2DM) and obesity are occurring with increasing frequency, and the incidence of GDM reflects this trend.

Objective: The aim of the study was to determine the relationship between first trimester elevated uric acid levels and subsequent development of gestational diabetes mellitus during pregnancy.

Patients and Methods: The study included 78 pregnant women in the first trimester attending the outpatient clinic of Zagazig University Hospitals, Zagazig for antenatal care. In this study, patients were classified according to their serum uric acid level into 3 groups.

Results: Women's GDM in group (I) showed that 3 (11.5\%) were normal, $16(61.5 \%)$ their fasting blood sugar levels (FBS) were high in $2^{\text {nd }}$ trimester and $7(26.9 \%)$ their FBS were high in both $2^{\text {nd }}$ and $3^{\text {rd }}$ trimesters while in group (II) $1(3.8 \%)$ were normal, $9(34.6 \%)$ their FBS were high in $2^{\text {nd }}$ trimester and $16(61.5 \%)$ their FBS were high in both $2^{\text {nd }}$ and $3^{\text {rd }}$ trimesters and in group (III) $1(3.8 \%)$ was normal, $5(19.2 \%)$ their FBS were high in $2^{\text {nd }}$ trimester and $20(76.9 \%)$ their FBS were high in both $2^{\text {nd }}$ and $3^{\text {rd }}$ trimesters. There was statistically significant differences between groups where $\mathrm{P}=0.007$.

Conclusion: First trimester serum uric acid levels are associated with subsequent development of IGT and GDM. The test has good predictive value for the diagnosis of GDM and it can be used in a risk assessment model.

Keywords: Gestational Diabetes Mellitus, Uric Acid, First Trimester of Pregnancy
\end{abstract}

\section{INTRODUCTION}

Gestational diabetes mellitus (GDM) is a common condition with risks for mother and baby. Type 2 diabetes mellitus (T2DM) and obesity are occurring with increasing frequency, and the incidence of GDM reflects this trend. Further, new diagnostic criteria have greatly increased the prevalence of GDM. Potential lifelong consequences exist for mother and baby if the condition goes untreated; however, effective pregnancy management can ameliorate adverse maternal and fetal effects ${ }^{(\mathbf{1})}$.

Pregnancies affected by GDM pose a risk for adversities such as the need for Caesarean sections due to fetal macrosomia. Macrosomia occurs because of accelerated fetal growth fueled by maternal hyperglycemia. In approximately $95 \%$ of GDM cases, maternal glucose metabolism returns to normal after delivery of the baby, however, an association between GDM and the development of diabetes mellitus in the mother later in life exists. In addition, research into the long-term effects of poor maternal glucose metabolism on the fetus has revealed that offspring born to mothers with GDM are susceptible to impaired glucose tolerance (IGT) and obesity. With these associations in mind, it would be important to identify pregnant women at risk for GDM so that prevention management such as lifestyle modifications can be implemented ${ }^{(2-4)}$.

Hypoglycemia in the neonatal period is another adverse effect of fetal hyperinsulinemia.
After birth, the infant is no longer exposed to the maternal source of glucose yet still has elevated circulating insulin levels, leading to hypoglycemia in the early neonatal period. Neonatal hypoglycemia must be addressed rapidly to minimize the risk of serious adverse consequences such as seizures, brain injury, and long-term neurodevelopmental impairment. Other adverse outcomes include hyperbilirubinemia and hypocalcemia, both of which may require close monitoring in the NICU ${ }^{(5)}$.

Additionally, the hyperglycemic intrauterine environment predisposes the fetus to early onset diabetes, in adolescence or early adulthood, even in populations with a relatively low incidence of T2DM. T2DM is a metabolic disease that is characterized by dysfunction in insulin secretion, insulin action, or both. This dysfunction leads to chronic insulin resistance and resulting chronic hyperglycemia. Typically, T2DM has an onset in adulthood however, with increasing prevalence of risk factors; early onset T2DM is becoming more common ${ }^{(6)}$.

The maternal consequences of GDM include preeclampsia and cesarean section in the short term and increased risk of T2DM in the long term. If GDM remains untreated, mothers are at a higher risk of preeclampsia and cesarean section. While GDM itself is not an indication for cesarean section, the complications associated with GDM, such as preeclampsia and macrosomia, may be an indication for cesarean section (C-section). If the mother is diagnosed with preeclampsia that necessitates early 
delivery and the mother's cervix has not sufficiently ripened for delivery, a C-section is indicated. Csection is indicated for macrosomia if the estimated fetal weight exceeds $4500 \mathrm{~g}$ in order to prevent shoulder dystocia and birth trauma during vaginal delivery ${ }^{(7)}$.

In order to manage the potential adverse outcomes of GDM, a mother's blood glucose must be controlled throughout her pregnancy. In all women with GDM, self-monitoring of blood glucose is recommended with the following goals based on American Obstetrics and Gynecology Guidelines (ACOG) - Fasting value: $<95 \mathrm{mg} / \mathrm{dL}, 1$ hour postprandial: <120-130 mg/dL, 2 hour postprandial: $<120 \mathrm{mg} / \mathrm{dL}$._When a woman presents with GDM, first line treatment to achieve euglycemia includes diet and exercise ${ }^{(\mathbf{8})}$.

If blood glucose levels remain uncontrolled with diet and exercise, pharmacologic treatment, such as insulin or oral hypoglycemic agents are used. Although oral hypoglycemic agents such as glyburide and metformin are commonly used as first-line pharmacologic therapy, the U.S. Food and Drug Administration have not approved them for GDM. Glyburide works by stimulating insulin production and release from the pancreas and is effective in lowering HbAlc concentrations, decreasing rates of macrosomia, and decreasing rates of neonatal hypoglycemia. Metformin is an insulin sensitizer in the liver and in the periphery and has been shown to be effective in decreasing adverse pregnancy outcomes. GDM can also be effectively managed with a combination of intermediate acting insulin preparations, such as neutral protamine Hagedorn (NPH), and short-acting insulin analogues, such as insulin lispro and insulin aspart (9).

Prediction and diagnosis of GDM is important for ongoing pregnancy and has important implications for subsequent health of the mother. GDM is considered a significant risk factor for subsequent development of type II diabetes and is associated with a poorer cardiovascular risk profile compared with women without GDM ${ }^{(10)}$.

The recommendation was based upon the inefficiency of the current history-based risk assessment method. However, the benefits of broad screening have not yet been established. A recent study by Koivunen et al. ${ }^{(11)}$ reported no benefit of broad screening on cesarean section rates and birth weight despite increased rates of GDM diagnosis, glucose-challenge test applications, and labor induction. Until the benefits of broad screening are established there is a need of a better risk assessment method. Uric acid has been investigated as a possible risk factor for the development of GDM. Several researchers reported an association of uric acid levels with development of GDM ${ }^{(\mathbf{1 2})}$.

\section{AIM OF THE WORK}

The aim of the study was to determine the relationship between first trimester elevated uric acid levels and subsequent development of gestational diabetes mellitus during pregnancy.

\section{PATIENTS AND METHODS}

Patients: The study included 78 pregnant women in the first trimester attending the outpatient clinic of Zagazig University Hospitals, Zagazig for antenatal care.

Patients' selection criteria: In order to be included in the study the women should have a normal fasting blood sugar level (70-100 mg/dl).

All patients included in the study fulfilled the following criteria:

\section{Exclusion Criteria:}

A) In the $1^{\text {st }}$ trimester (13 - 15 weeks).

B) Singleton pregnancy.

C) Age 18-35 years.

D) Body mass index $=$ weight in $\mathrm{kg}$ /height in $\mathrm{m}^{2}$ (18.5 - 30).

- Patient with past history of gestational diabetes.

- Renal disease.

- Tuberculosis.

- Bronchial asthma.

- Liver disease.

- Cardiovascular disease.

- Gout.

- Connective tissue disorder.

- Chronic hypertension.

- Woman on drugs causing hyperuricemia. (e.g. ethambutol nicotinic acid, salicylates, and pyrazinamide)

- Obese women (Body mass index > 30).

- Multiple pregnancy.

Type of study: prospective observational clinical study.

Patients were classified according to their serum uric acid level into 3 groups:

- Group (A): 26 women with uric acid $<3 \mathrm{mg} / \mathrm{dl}$.

- Group (B): 26 women with uric acid from 3-4 $\mathrm{mg} / \mathrm{dl}$.

- Group (C): 26 women with uric acid $>4 \mathrm{mg} / \mathrm{dl}$.

The samples were collected in 3 visits as follows:

- In the $1^{\text {st }}$ visit (antenatal visit), where uric acid and fasting blood sugar was tested.

- In the $2^{\text {nd }}$ visit which was taking place during the $24-28$ weeks of gestational age, where fasting and 2 hours post prandial blood sugar was tested.

- In the $3^{\text {rd }}$ visit which was taking place during the 34-36 weeks of gestational age, where fasting and 2 hours post prandial blood sugar was tested. 
Methods:

Technical design:

- Study setting: This study was carried out at Obstetrics and Gynecology Outpatient and Department in Zagazig University Hospitals.

- Sample size: By assuming that the percentage (\%) is $50 \%$, and the cutoff serum uric acid (UA) is 3.6 $\mathrm{mg} / \mathrm{dl}$; when UA level is $>3.6 \mathrm{mg} / \mathrm{dl}$ developed (GDM) and the mean age of the sample is 27.3 and the Standard Deviation (SD) is 2.2, with Confidence Level of 95\% CI, and power of test equals $80 \%$ and by using EPI info. Version 6. Sample size is calculated to be 78 antenatal female patients less than 14 weeks of gestational age.

Tools: An informed consent was taken from patients. In case of incompetent patients, the informed consent was taken from the guardians.

The scientific and ethical approval was also obtained at the Faculty of Medicine, AlAzhar University.

Following the informed consent, all patients were subjected to the following:

\section{All cases were subjected in the first visit to:}

\section{History taking:}

Detailed history taking regarding,

- Personal history: Name, age, occupation, residence.

- Menstrual history: Last menstrual period (LMP), regularity

- Obstetric history: Gravidity, parity, mode of previous delivery, pre-eclampsia or gestational diabetes in any previous pregnancies.

- Past history: Medical disease especially hypertensive disorders, diabetes and surgical.

\section{Clinical examination:}

I. General examination: including:

1.Vital signs including blood pressure in $\mathrm{mmHg}$, pulse, temperature.

2. Weight, height then we calculate body mass index (BMI).

3. Chest and heart examination.

4.Oedema.

\section{Obstetric examination:}

- Abdominal examination. (Including routine examination and ultrasound for pregnancy).

\section{Lab investigations:}

\section{In the first visit in the $1^{\text {st }}$ trimester:}

All patients included in the study were subjected to: Routine investigations of early pregnancy including (Hemoglobin $(\mathrm{Hb})$ in $\mathrm{gm} / \mathrm{dl}$, Rhesus factor (Rh) typing, fasting blood sugar levels (FBS) in $\mathrm{mg} / \mathrm{dl}$, complete urine analysis). Serum uric acid concentration in $\mathrm{mg} / \mathrm{dl}$.

\section{In the second and third trimesters:}

At 24 to 28 weeks of gestation, all participants were instructed to fast for at least 8 hours before sampling. A fasting glucose sample was taken. This sample was collected for the fasting plasma glucose (FPG) value. After intake of the $75 \mathrm{~g}$ glucose load, blood samples were drawn 60 and 120 min later for the determination of glucose levels.

Diagnostic criteria and classification of GDM by WHO, 2013 states that GDM should be diagnosed if one or more of the following criteria are met or exceeded:

a) Fasting plasma glucose $(92 \mathrm{mg} / \mathrm{dL})$.

b) 1-hour plasma glucose $(180 \mathrm{mg} / \mathrm{dL})$ following a $75 \mathrm{~g}$ oral glucose load.

c) 2-hour plasma glucose $(153 \mathrm{mg} / \mathrm{dL})$ following a $75 \mathrm{~g}$ oral glucose load.

If the values were within normal range, the tests were repeated again at 34-36 weeks.

Confirmatory tests: Positive cases with glucose challenge test were confirmed using:

\section{OGTT 3 hours' glucose tolerance curve:}

The 3 hour OGTT is a diagnostic test for gestational diabetes. It measures glucose concentrations over three hours after a 100 -g oral glucose load. The test was done in the morning after an overnight fasting of $8 \mathrm{~h}$, and after at least 3 days of unrestricted diet and physical activity. The diagnosis of GDM was made if at least two of the following four plasma glucose levels (measured fasting and $1 \mathrm{~h}, 2 \mathrm{~h}, 3 \mathrm{~h}$ during OGTT) are met or exceeded:

\begin{tabular}{|c|c|}
\hline Fasting & $95 \mathrm{mg}$ \\
\hline $1 \mathrm{~h}$ & $180 \mathrm{mg}$ \\
\hline $2 \mathrm{~h}$ & $155 \mathrm{mg}$ \\
\hline $3 \mathrm{~h}$ & $140 \mathrm{mg}$ \\
\hline
\end{tabular}

\section{Statistical analysis}

In addition to the descriptive data, statistical analysis was done using IBM SPSS STATISTC VERSION 23 PROGRAM. Data were expressed as mean \pm SD and analyzed using the Student's t-test, Paired $(t)$ test and ANOVA test to assess the significance of difference in the levels between different parameters. $\mathrm{P}<0.05$ was accepted as significant.

Coefficient ( $\mathrm{r}$ ) of two variables was also done by using Pearson Correlation Coefficient (r) with P Value Calculation.

\section{RESULTS}

$1^{\text {st }}$ trimester: At gestational age 13 - 15 weeks:

Regarding age, there was no statistically significant differences between groups (Table 1). 
Table (1): Comparison between three groups as regard to patient's age (years)

\begin{tabular}{||c|c|c|c||}
\hline Age & Group (I) & Group (II) & Group (III) \\
\hline $\begin{array}{c}\text { Min.- } \\
\text { Max. }\end{array}$ & $19-33$ & $19-35$ & $18-35$ \\
\hline $\begin{array}{c}\text { Mean } \pm \\
\text { S.D }\end{array}$ & $25.08 \pm 4.298$ & $27.42 \pm 4.933$ & $27.08 \pm 5.782$ \\
\hline $\begin{array}{c}\text { P } \\
\text { Value }\end{array}$ & \multicolumn{3}{|c|}{0.201} \\
\hline
\end{tabular}

Regarding gestation age, there was no statistically significant differences between groups (Table 2).
Table (2): Comparison between three groups as regard to patient's gestational age 13-15 weeks

\begin{tabular}{|c|c|c|c|}
\hline $\begin{array}{c}\text { Gestation } \\
\text { al Age }\end{array}$ & Group (I) & Group (II) & $\begin{array}{c}\text { Group } \\
\text { (III) }\end{array}$ \\
\hline Min.-Max. & $13-15$ & $13-15$ & $13-15$ \\
\hline $\begin{array}{c}\text { Mean } \pm \\
\text { S.D }\end{array}$ & $\begin{array}{c}13.46 \pm 0.64 \\
7\end{array}$ & $13.50 \pm 0.58$ & $13.50 \pm 0.58$ \\
\hline P Value & \multicolumn{3}{|c|}{0.966} \\
\hline
\end{tabular}

As for women's height, weight and BMI, there were no statistically significant differences between groups (Table 3).

Table (3): Comparison between three groups as regard to height, weight and BMI at gestational age 13-15 weeks

\begin{tabular}{|c|c|c|c|c|}
\hline & Group (I) & $\overline{\text { Group (II) }}$ & $\overline{\text { Group (III) }}$ & $\overline{P \text { P-Value }}$ \\
\hline \multicolumn{5}{|l|}{ Height } \\
\hline Min. - Max. & $150-169$ & $157-173$ & $156-169$ & \multirow{2}{*}{0.269} \\
\hline Mean \pm S.D & $163.77 \pm 4.811$ & $165.35 \pm 3.999$ & $163.62 \pm 3.930$ & \\
\hline \multicolumn{5}{|l|}{ Weight } \\
\hline Min. - Max. & $52-90$ & $53-85$ & $53-69$ & \multirow{2}{*}{0.981} \\
\hline Mean \pm S.D & $62.04 \pm 7.587$ & $62.00 \pm 6.928$ & $61.12 \pm 4.719$ & \\
\hline \multicolumn{5}{|l|}{ BMI } \\
\hline Min. - Max. & $19.61-32.66$ & $20.20-28.40$ & $20.20-27.53$ & \multirow{2}{*}{0.922} \\
\hline Mean \pm S.D & $23.162 \pm 2.873$ & $22.666 \pm 2.210$ & $22.847 \pm 1.825$ & \\
\hline
\end{tabular}

As regard to the women's FBS, there were statistically significant differences between groups (Table 4).

Table (4): Comparison between three groups as regard to patient's FBS at gestational age 13-15 weeks

\begin{tabular}{|c|c|c|c|}
\hline FBS & Group (I) & Group (II) & Group (III) \\
\hline Min. - Max. & $84-130$ & $90-137$ & $95-142$ \\
\hline Mean \pm S.D & $95.12 \pm 11.392$ & $108.50 \pm 10.779$ & $121.69 \pm 12.927$ \\
\hline P Value & & $0.001^{*}$ \\
\hline
\end{tabular}

At gestational age $24-28$ weeks:

As regard to the women's FBS, there were statistically significant differences between groups (Table 5).

Table (5): Comparison between three groups as regard to patient's FBS at gestational age 24-28 weeks

\begin{tabular}{||c|c|c|c|}
\hline FBS & Group (I) & Group (II) & Group (III) \\
\hline$<\mathbf{1 0 0}$ & $3(11.5 \%)$ & $1(3.8 \%)$ & $1(3.8 \%)$ \\
\hline$>\mathbf{1 0 0}$ & $23(88.5 \%)$ & $25(96.2 \%)$ & $25(96.2 \%)$ \\
\hline Min. - Max. & $95-139$ & $96-150$ & $100-150$ \\
\hline Mean \pm S.D & $119.42 \pm 14.437$ & $137 \pm 13.005$ & $136.46 \pm 12.481$ \\
\hline P Value & \multicolumn{3}{|c|}{$0.001 *$} \\
\hline
\end{tabular}

As for women's postprandial test at $1^{\text {st }}$ hour, at $2^{\text {nd }}$ hour, and at $3^{\text {rd }}$ hour in group; there were no statistically significant differences between groups (Table 6). 
Ali El- Shabrawy et al.

Table (6): Comparison between three groups as regard to patient's postprandial test at gestational age 24-28 weeks

\begin{tabular}{|c|c|c|c|}
\hline Postprandial & $\mathbf{1}^{\text {st }}$ hour & $2^{\text {nd }}$ hour & $\mathbf{3}^{\text {rd }}$ hour \\
\hline \multicolumn{4}{|l|}{ Group (I) $(n=23)$} \\
\hline Min. - Max. & $181-200$ & $158-185$ & $140-165$ \\
\hline Mean \pm S.D & $190.391 \pm 6.051$ & $171.435 \pm 8.994$ & $151.826 \pm 7.505$ \\
\hline \multicolumn{4}{|l|}{ Group (II) (n=25) } \\
\hline Min. - Max. & $180-200$ & $156-185$ & $140-162$ \\
\hline Mean \pm S.D & $190.92 \pm 5.522$ & $170.92 \pm 9.133$ & $150.800 \pm 6.062$ \\
\hline \multicolumn{4}{|l|}{ Group (III) $(\mathrm{n}=25)$} \\
\hline - $\quad$ Min. - Max. & $181-200$ & $155-185$ & $140-165$ \\
\hline Mean \pm S.D & $190.84 \pm 5.850$ & $170.080 \pm 10.128$ & $153.520 \pm 7.054$ \\
\hline P value & 0.945 & 0.881 & 0.375 \\
\hline
\end{tabular}

$3^{\text {rd }}$ trimester: At gestational age 34 - 36 weeks:

Regarding to women's FBS, there were statistically significant differences between groups (Table 7).

Table (7): Comparison between three groups as regard to patient's FBS at gestational age 34-36 weeks

\begin{tabular}{||c|c|c|c|}
\hline FBS & Group (I) & Group (II) & Group (III) \\
\hline$<\mathbf{1 0 0}$ & $19(73.1 \%)$ & $10(38.5 \%)$ & $5(19.2 \%)$ \\
\hline$>\mathbf{1 0 0}$ & $7(26.9 \%)$ & $16(61.5 \%)$ & $21(80.8 \%)$ \\
\hline Min. - Max. & $95-128$ & $95-139$ & $95-140$ \\
\hline Mean \pm S.D & $102.54 \pm 9.626$ & $115.62 \pm 16.589$ & $119.92 \pm 14.800$ \\
\hline P Value & \multicolumn{3}{|c}{$0.001 *$} \\
\hline
\end{tabular}

As regard to women's postprandial test at $1^{\text {st }}$ hour, at $2^{\text {nd }}$ hour, and at $3^{\text {rd }}$ hour in group; there were no statistically significant differences between groups (Table 8).

Table (8): Comparison between three groups as regard to patient's postprandial test at gestational age 34-36 weeks

\begin{tabular}{||c|c|c|c|}
\hline Postprandial & $\mathbf{1}^{\text {st }} \mathbf{h o u r}$ & $\mathbf{2}^{\text {nd }}$ hour & $\mathbf{3}^{\text {rd }}$ hour \\
\hline Group (I) (n=7) & & & \\
\hline Min. - Max. & $151-188$ & $120-187$ & $120-144$ \\
\hline Mean \pm S.D & $169.143 \pm 15.614$ & $144.571 \pm 21.915$ & $128.857 \pm 8.840$ \\
\hline Group (II) (n=16) & & & $120-152$ \\
\hline Min. - Max. & $151-185$ & $120-164$ & $130.125 \pm 9.479$ \\
\hline Mean \pm S.D & $165.125 \pm 9.818$ & $138.625 \pm 13.525$ & $120-159$ \\
\hline Group (III) (n=21) & & & $132.429 \pm 11.138$ \\
\hline Min. - Max. & $150-187$ & $125-177$ & 0.662 \\
\hline Mean \pm S.D & $169.095 \pm 11.484$ & $149.619 \pm 14.988$ & 0.121 \\
\hline P value & 0.555 & & \\
\hline
\end{tabular}

Women's GDM in in the 3 groups showed statistically significant differences (Table 9).

Table (9): Comparison between three groups as regard to patient's GDM at gestational age 34-36 weeks

\begin{tabular}{|c|c|c|c|c|c|c|}
\hline GDM & \multicolumn{2}{|c|}{ Group (I) } & \multicolumn{2}{|c|}{ Group (II) } & \multicolumn{2}{|c|}{ Group (III) } \\
\hline Normal & 3 & 11.5 & 1 & 3.8 & 1 & 3.8 \\
\hline High in $2^{\text {nd }}$ trimester & 16 & 61.5 & 9 & 34.6 & 5 & 19.2 \\
\hline High in $2^{\text {nd }}$ and $3^{\text {rd }}$ trimester & 7 & 26.9 & 16 & 61.5 & 20 & 76.9 \\
\hline P Value & \multicolumn{6}{|c|}{$0.007 *$} \\
\hline
\end{tabular}




\section{DISCUSSION}

In this study, Patients were classified according to their serum uric acid level into 3 groups: Group (I): 26 women with uric acid $<3 \mathrm{mg} / \mathrm{dl}$. Group (II): 26 women with uric acid from $3-4 \mathrm{mg} / \mathrm{dl}$. Group (III): 26 women with uric acid $>4 \mathrm{mg} / \mathrm{dl}$.

Women's age in group (I) ranged between 19 - 33 years with mean \pm SD $25.08 \pm 4.298$ years while in group (II) ranged between $19-33$ years with mean \pm SD $27.42 \pm 4.933$ years and in group (III) ranged between 18 - 35 years with mean \pm SD $27.08 \pm 5.782$ years. There was no statistically significant differences between the groups. Women's gestational age in group (I) ranged between $13-15$ weeks with mean \pm SD $13.46 \pm 0.647$ weeks while in group (II) ranged between $13-15$ weeks with mean \pm SD $13.50 \pm 0.583$ weeks and in group (III) ranged between 13 - 15 weeks with mean \pm SD $13.50 \pm 0.583$ weeks. There was no statistically significant differences between the groups.

This slight increase in mean age could be due to our changing trend of getting married late or due to delay in begetting child. Incidence of GDM in the present study is quite high $(22.6 \%)$ than those reported by past studies for India (1-14\%). However Seshiahet al. ${ }^{(13)}$ reported age based incidence of GDM among women, as up to $15 \%$ in women of 30 years.

In another study Choi et al. ${ }^{(14)}$ found that uric acid in the first trimester likely approximates pre-conception uric acid, and elevated uric acid may identify women who are predisposed to metabolic syndrome with an increased risk of developing GDM, independent of obesity.

Alternatively, uric acid decreases early in pregnancy, so perhaps women with elevated uric acid decreases early in pregnancy, so perhaps women with elevated uric acid have a poor adaptation to pregnancy (i.e., abnormal placentation), putting them at risk for adverse pregnancy outcomes such as GDN ${ }^{(\mathbf{1 4})}$.

Our results are in contrast to Gungor $\boldsymbol{e t}$ al. (15), who compared the relationship between serum uric acid, creatinine and albumin levels in pregnant women with normal glucose tolerance and gestational diabetes mellitus. A total of 112 patients were evaluated, 56 of whom had gestational diabetes. All of the patients had single estimations of serum uric acid, creatinine, albumin and liver enzymes carried out on booking between 24-28 weeks gestation. The women were followed up throughout pregnancy. They found that single estimations of serum uric acid levels were higher in the diabetic patients, but this elevation was not statistically significant and also albumin concentrations were not significantly different between a normal pregnant group and a GDM group. Gungor et $\boldsymbol{a l} .{ }^{(15)}$ results were in contrary to the findings of our study, this may be due to the differences in the number of cases and gestational age of serum uric acid estimations.

In our study the pregnancies were categorized according to maternal age, into 6 categories, >20 years, 20-24 years, 25-29 years, 3034 years, 35-39 years and <years. (96.6\%) patients continued their pregnancies beyond the first trimester, and the number (percentage of total) from the youngest to the oldest cohort were $318(2.0 \%)$, 1.713 (10.8\%), 4.446 (28.1\%), 5.457 (34.5\%), 3.279 (20.7\%) and 614 (3.9\%), respectively. Correlation in the prevalence of GDM, was increasing from 1.3, $2.5,6.2,21.7$ and $31.9 \%$, respectively from the youngest to the oldest cohort $(\mathrm{P}>0.001)$. The risk for the older cohorts was significantly increased as follows: 25-29 years, 2.59 (1.84-3.67), 30-40 years, 4.38 (3.13-6.13), 35-39 years, 10.85 (7.72- 15.25) and <years, 15.90 (10.62-23.80).

There was no significant difference for the $>20$ years, $10.85(7.72-15.25)$ and <years 15.90 (10.62-23.80). There was no significant difference for the $>20$ years cohorts. There finding indicates that the risk of GDM becomes significantly and progressively increased from 25 years onwards. This supports the American Diabetes Association recommendation on the use of age 25 years as the cutoff for screening and the observation that maternal age 25 years is factors most predictive of GDM ${ }^{(\mathbf{1 6})}$.

In our study, the mean BMI was significantly higher in women who developed GDM when compared to women who did not develop GDM (Normal 115 cases of total sample and no one developed GD with mean+SD, 95.57+12.32, overweight 59 cases, 56 cases $(29.3 \%)$ were normal and 3 cases $(33.3 \%)$ had GD with mean+SD $106.29+26.62$, obese 26 cases, 20 cases $(10.5 \%)$ were normal and 6 cases $(66.7 \%)$ had GD with mean+SD 124.27+39.78, p-value 0.000). This agrees with Loa et al. ${ }^{\left({ }^{16)}\right.}$ who studied 1121 patients with GDM who were referred to the outpatient clinic of Szczecin University in Poland between January 2001 and December 2005. The control group consisted of 1011 healthy pregnant women. All had singleton pregnancies. Significant relationships between pregravid BMI and GDM treated with insulin. This study states the same results as our study. In another study, Jenny et al. ${ }^{(17)}$ who studied 1733 patients with singleton pregnancies enrolled in project viva. They examined the associations of first trimester diet, with results of glucose tolerance testing at 26-28 week's gestation. 91 patients developed GDM and 206 patients had impaired glucose tolerance (IGT). They concluded that pre- 
pregnancy body mass index (BMI) is a strong predictor for development of GDM ${ }^{(17)}$.

Hyperuricemia has been associated with increasing body mass index (BMI) in several studies and are even apparent in the adolescent youth ${ }^{(18)}$.

These studies Loa et al. ${ }^{\left({ }^{(16)}\right.}$ and Jenny $\boldsymbol{e t}$ al. (17) stated the same results as our study. Hyperuricemia may also be present in the metabolic syndrome in people who are not overweight or obese. In one study only $5.9 \%$ of subjects with a normal BMI and a uric acid level of less than $6.0 \mathrm{mg}$ per deciliter had the metabolic syndrome; in contrast, $59 \%$ of subjects with a normal BMI and a uric acid level of more than $1.0 \mathrm{mg}$ per deciliter had evidence of the metabolic syndrome. These are against the findings of our study. This may be due to differences in technique, time and sample size ${ }^{(\mathbf{1 0})}$.

In this present study, serum uric acid at recruitment was insignificantly associated with a fasting blood glucose $\geq 105 \mathrm{mg} / \mathrm{dl}$ among women who developed GDM [OR 0.66, 95\% CI (0.395 to $0.57)]$. In a large cross sectional study of 53,477 non- pregnant adults, serum uric acid was positively correlated with fasting serum glucose $\geq 110$ $\mathrm{mg} / \mathrm{dl}^{\left(\mathbf{1 7}^{17}\right)}$. The results of this study were against ours.

In this study, women's uric acid showed statistically significant differences between groups. In another study, In Queen Mary Hospital, the University of Hong Kong ${ }^{(\mathbf{1 9})}$.

Have reviewed the prevalence of GDM, diagnosed by the World Health Organization criteria in over 15000 singleton pregnancies managed from 1998 to 2001. These finding indicates that the risk of GDM becomes significantly and progressively increased from 25 years onwards. This supports the American Diabetes Association recommendation on the use of age 25 years as the cutoff for screening and the observation that maternal age 25 years is the factor most predictive of GDM. The results of this study were coincides with our result ${ }^{(20)}$.

In this study, regarding women's FBS there were statistically significant differences between groups. In a nested case-control study by, KautzkyWiller et al. (21) 22 women with GDM, 10 with IGT, and 20 healthy pregnant women were chosen from the women who had visited at 14-20 weeks of gestation and had blood samples prospectively taken and kept during their visit. Findings of this study showed that women with GDM have the highest values of leptin compared with those with IGT and the healthy controls at $14-20$ weeks of gestation. Some studies evaluated the relationship between leptin concentration and insulin resistance. Maghbooli et al. ${ }^{(22)}$ found that leptin concentration was positively associated with insulin level and HOMA index. Liu et al. ${ }^{(23)}$ showed a positive and significant correlation between the maternal leptin and fasting insulin levels. Our results indicate that plasma insulin levels are higher in GDM and IGT compared with the normal group, and the serum leptin had a significant positive correlation with insulin and HOMA index. However, Mohiti $\boldsymbol{e t}$ al. (24) reported that the serum leptin had a negative correlation with insulin in obese diabetic patients.

Our findings are consistent with a much larger body of evidence from experimental, clinical, and epidemiological investigations, which suggest that leptin is an important mediator of glucose homeostasis in humans and plasma leptin has a significant positive correlation with BMI. Liu et al. (23) also has shown that leptin predicts the development of GDM independent of maternal BMI and other risk factors. The findings of this prospective study are generally consistent with the reports by Lappas et al. ${ }^{(25)}$ and Qiu $\boldsymbol{e t}$ al. ${ }^{(26)}$, as well. Our finding showed that the predicting threshold for GDM was serum leptin levels 20.5 $\mathrm{ng} / \mathrm{mL}$, but as the area under the curve is less than $80 \%$, this threshold is not the best predicting point for leptin in GDM women. So, we need a prospective study in larger population of GDM women. Patients with GDM had higher serum leptin levels than IGT and non-GDM women, and women with higher BMI had a higher level of leptin concentration. These results show the presence of an association between serum leptin level and glucose metabolism in GDM. As to whether the high leptin levels can serve as a predictive factor for GDM development, larger prospective studies are needed to address this issue.

In this study, women's postprandial test at $1^{\text {st }}$ hour, at $2^{\text {nd }}$ hour, and at $3^{\text {rd }}$ hour in group; showed no statistically significant differences between groups. Hollinworth and Grundy ${ }^{(27)}$ even observed a lower plasma level of LDL-C in women with gestational diabetes mellitus.

Also the influence of genetic and environmental factors on endogenous pathway of lipids cannot be completely ruled out. This may affect endogenous synthesis of cholesterol that may have an overall influence on lipid metabolism in pregnant and non-pregnant women as well in the disease state ${ }^{(27)}$.

In this study, women's GDM in group (I) show that $3(11.5 \%)$ were normal, $16(61.5 \%)$ their FBS were high in $2^{\text {nd }}$ trimester and 7 (26.9\%) their FBS were high in both $2^{\text {nd }}$ and $3^{\text {rd }}$ trimesters while in group (II) 1 (3.8\%) were normal, 9 (34.6\%) their FBS were high in $2^{\text {nd }}$ trimester and $16(61.5 \%)$ their FBS were high in both $2^{\text {nd }}$ and $3^{\text {rd }}$ trimesters and in group (III) $1(3.8 \%)$ were normal, $5(19.2 \%)$ their FBS were high in $2^{\text {nd }}$ trimester and $20(76.9 \%)$ their FBS were high in both $2^{\text {nd }}$ and $3^{\text {rd }}$ trimesters. There was statistically significant differences between groups. 
In another study, GDM was the most common metabolic disorder complicating pregnancy. Approximately 3-5\% of all pregnancies are complicated by diabetes and $90 \%$ of these are GDM and rest is pregestational. Study by Deborah L. Conway shows an incidence of 2-5\%. Deborah (28) our study included 134 GDM patient's incidence being 2.4\%. According to Hold et al. ${ }^{(29)}$ GDM is linked to several maternal, fetal and neonatal complications the characteristics of which are same is that of pregestational diabetes ${ }^{(30)}$.

\section{CONCLUSION AND RECOMMENDATIONS}

- First trimester serum uric acid levels are associated with subsequent development of IGT and GDM. The test has good predictive value for the diagnosis of GDM and it can be used in a risk assessment model and screening of GDM at 2428 weeks gestation.

- The cutoff level of maternal serum uric acid of 4 $\mathrm{mg} / \mathrm{dl}$ in the first trimester was associated with developing GDM. Therefore, we recommend that serum uric acid level should be done as a routine test during the first antenatal care visit, but large scale studies are required to support this recommendation.

\section{REFERENCES}

1. Herring SJ, Oken E (2011): Obesity and diabetes in mothers and their children: can we stop the intergenerational cycle? Curr Diab Rep., 11: 20-27

2. Duan T, Yang H, Van Assche A et al. (2015): The International Federation of Gynecology and Obstetrics (FIGO) Initiative on gestational diabetes mellitus: A pragmatic guide for diagnosis, management, and care\#. Int $\mathbf{J}$ Gynecol Obstet., 131(3):173-211.

3. Popkin BM, Adair LS and Ng SW (2012): Global nutrition transition and the pandemic of obesity in developing countries. Nutr Rev., 70: 3-21

4. American Diabetes Association (2014): Standards of medical care in diabetes2014. Diabetes Care, 37(1):14-80.

5. The International Diabetes Federation (2013): IDF Diabetes Atlas Sixth Edition. https://www.idf.org/e-library/epidemiology-

research/diabetes-atlas.html

6. Hoy D, Brooks P, Woolf A et al. (2012): Assessing risk of bias in prevalence studies: modification of an existing tool and evidence of interrater agreement. J Clin Epidemiol., 65: 934-939.

7. Kuti MA, Abbiyesuku FM, Akinlade KS et al. (2011): Oral glucose tolerance testing outcomes among women at high risk for gestational diabetes mellitus. J Clin Pathol., 64: 718-721.

8. Metzger BE, Gabbe SG, Persson B et al. (2010): International association of diabetes and pregnancy study groups recommendations on the diagnosis and classification of hyperglycemia in pregnancy. Diabetes Care, 33: 676-682

9. Hirst JE, Raynes-Greenow CH and Jefferey HE (2012): A systematic review of trends of gestational diabetes mellitus in Asia. J Diabetology, 3: 1-12.

10. Moon JH, Kwak SH, Jung HS et al. (2015): Weight Gain and Progression to Type 2 Diabetes in Women with a History of Gestational Diabetes Mellitus. J Clin Endocrinol Metab., 100: 3548-55.

11. Koivunen S, Kajantie E, Torkki A et al. (2015): The changing face of gestational diabetes: The effect of the shift from risk factor-based to comprehensive screening. Eur J Endocrinol., 173: 623-32.

12. Güngör ES, Danişman N, and Mollamahmutoğlu L (2006): Relationship between serum uric acid, creatinine, albumin and gestational diabetes mellitus. Clin Chem Lab Med., 44:974-7.

13. Seshiah V, Das AK, Balaji V et al. (2006): Gestational diabetes mellitus-guidelines. JAPI., 54:622-8.

14. Choi HK, Curhan G (2007): Independent impact of gout on mortality and risk for coronary heart disease. Circulation, 116: 894-900.

15. Gungor ES, Danisman Mollamahmutoglu $\mathbf{L}$ (2006): Relationship between serum uric acid, creatinine, albumin and gestational diabetes mellitus. Clin Chem Lab Med., 44: 974-977.

16. Loa MD, Bolnick J and Cloakey D (2003): The use of glyburide in the management of gestational diabetes. Obstet Gynecol., 88:193-205.

17. Jenny SR, Emily O, Sheryl LR et al. (2008): Diet during early pregnancy and development of gestational diabetes. Paediatr Perinat Epidemiol., 22: 47-59.

18. Conen D, Wietlisbach V, Bovet $P$ et al. (2004): Prevalence of hyperuricemia and relation of serum uric acid with cardiovascular risk factors in a developing country. BMC Public Health, 4: 9-13.

19. Lao TT, Ho LF, Chan BC et al. (2006): Maternal age and prevalence of gestational diabetes mellitus. Diabetes Care, 29 (4): 948-9.

20. Ogonowski J, Miazgowski T, Kuczy'N et al. (2009): Pregravid body mass index as a predictor of gestational diabetes mellitus. Journal compilation Diabetes UK. Diabetic Medicine, 26: 334-8.

21. Kautzky-Willer A, Pacini G, Tura A et al. (2001): Increased plasma leptin in gestational diabetes. Dia-betologia J., 44:164

22. Maghbooli Z, Hossein-Nezhad A, Rahmani M et al. (2007): Relationship between leptin concentration and insulin resistance. Horm Metab Res., 39:903-7.

23. Liu ZJ, Liu PQ, Ding Y et al. (2003): Maternal plasma leptin levels and their relationship to insulin and glucose in pregnant women with gestational diabetes mellitus and gestational impaired glucose tolerance. Zhonghua Fu Chan Ke Za Zhi., 38:2613.

24. Mohiti J, Afkhami $M$ and Babaei $A$ (2005): Relation between leptin and insulin in 
patientswithtype II diabetesmellitus. Int $\mathrm{J}$ Endocrinol Metab., 3:121-5.

25. Lappas M, Yee K, Permezel M et al. (2005): Release and regulation of leptin, resistin and adiponectin from human placenta, fetal membrane, and maternal adipose tissue and skeletal muscle from normal and gestational diabetes mellitus complicated pregnancies. J Endocrinol., 186:45765.

26. Qiu C, Williams MA, Vadachkoria $S$ et al. (2004): Increased maternal plasma leptin in early pregnancy and risk of gestational diabetes mellitus. Obset Gynecol J., 103:519-25.
27. Hollinworth DR, Grundy SM (1982): Pregnancy associated hypertriglyc-eridemia in normal and diabetic women. Diabetes, 31: 1092-1097.

28. Deborah C (2014): Management of high risk pregnancy 5th edition. https://www.wiley.com/.../Management+of+Hig h+Risk+Pregnancy \%3A+An+Evidence..

29. Hold $M$, Merlob $P$ and Friedman $S$ (1999): Gestational diabetes mellitus; a survey of perinatal complication. Diabetes, 40 (2): 74-8.

30. Greene MF (1997): Screening for gestational diabetes. N Engl J med., 337: 1625-6. 